\title{
BMJ Open Comparison of Taiji and aerobic exercise for functional constipation: study protocol for a randomised controlled neuroimaging trial
}

\author{
Sha Yang, ${ }^{1}$ Jingwen Chen, ${ }^{1}$ Yuyi Guo, ${ }^{1}$ Yuke Teng, ${ }^{1}$ Tianyu Liu, ${ }^{2}$ Rongtao Ying, ${ }^{1}$ \\ Zhaoxuan He, ${ }^{1}$ Jianwei Wu, ${ }^{3}$ Shu-Guang Yu, ${ }^{4}$ Fang Zeng ${ }^{1}$
}

To cite: Yang S, Chen J, Guo Y, et al. Comparison of Taiji and aerobic exercise for functional constipation: study protocol for a randomised controlled neuroimaging trial. BMJ Open 2019;9:e031089. doi:10.1136/ bmjopen-2019-031089

- Prepublication history and additional material for this paper are available online. To view these files, please visit the journal online (http://dx.doi org/10.1136bmjopen-2019031089).

SY, JC and YG contributed equally.

SY, JC and YG are joint first authors.

Received 17 April 2019 Revised 26 July 2019 Accepted 29 July 2019

Check for updates

(C) Author(s) (or their employer(s)) 2019. Re-use permitted under CC BY-NC. No commercial re-use. See rights and permissions. Published by BMJ.

For numbered affiliations see end of article.

Correspondence to Professor Shu-Guang Yu; ysg@cdutcm.edu.cn

Professor Jianwei Wu; wujianwei@cdutcm.edu.cn

\section{ABSTRACT}

Introduction Taiji has been proven to be effective for regulating both the physical and mental state compared with simple aerobic exercise. However, whether the improvement of Taiji for constipation is related to regulate imbalanced brain-gut axis and emotional disorder for functional constipation (FC) remains uncertain. The results of the study will demonstrate the differences in regulation brain-gut balance between Taiji and simply aerobic exercise for patients with FC and provide a potential therapy for clinical treatment of FC, and a new approach for the research of mind-body exercise.

Methods and analysis In this randomised controlled neuroimaging trial, 80 patients with FC will be allocated into two groups: Taiji group and aerobic exercise group. The two groups will receive 10 weeks of Taiji exercise or aerobic exercise, respectively. The stool diary, Cleveland Constipation Score and Patient Assessment of Constipation Symptom, Patient Assessment of Constipation Quality of Life Questionnaire will be used to evaluate the clinical efficacy, the Self-rating Depression Scale, Self-rating Anxiety Scale, Eysenck Personality Questionnaires and Mini-Mental State Examinations will be used to assess the mental state at the baseline, the 5-week intervention and the end of intervention. The 24-hour heart rate variability will be used for assessing the autonomic nervous function, functional MRI and positron emission tomography-CT will be performed for detecting the cerebral functional changes at the baseline and the end of the intervention. The clinical data and multimodal imaging data will be analysed, respectively. Correlation analysis will be conducted to investigate the relationship between cerebral functional changes and symptom improvement.

Ethics and dissemination The procedures have been approved by the Sichuan Regional Ethics Review Committee on Traditional Chinese Medicine (No. 2018KL047) and conformed to the Declaration of Helsinki. Results will be disseminated through policy briefs, workshops, peer-reviewed publications and conferences.

Trial registration number Chinese Clinical Trial Registry (ChiCTR1800019781).

\section{INTRODUCTION}

Functional constipation (FC), a common functional gastrointestinal disorder (FGID),
Strengths and limitations of this study

- The use of randomised controlled neuroimaging trial design will provide valid evidence about the effectiveness of simple aerobic exercise and Taiji (mindbody exercises) for treating functional constipation (FC).

- This study is the first to investigate the differences in regulation brain-gut balance between Taiji and simply aerobic exercise for patients with FC.

- This study can provide a potential therapy for clinical treatment of FC, and a new approach to investigate mind-body exercises from clinical symptoms, mental state and brain function.

is characterised by infrequent defecation, hard or lumpy stools, straining during defecation, the sensation of anorectal obstruction/blockage and incomplete evacuation. ${ }^{1}$ The estimated global prevalence of FC ranges from $6 \%$ to $29.6 \% .^{2-5}$ In addition to the higher prevalence, FC significantly influences the patients' quality of life (QoL) and brings remarkable healthcare costs. ${ }^{6-8}$ Currently, pharmacotherapy for FC including cathartic is not satisfactory in improving persistent symptoms and the QoL of patients with FC. Besides, the side effects are often reported ${ }^{9}$ such as bloating, ${ }^{10}$ diarrhoea ${ }^{11}$ and nausea. ${ }^{12}$ Therefore, it is urgent to find safe and effective treatment for FC.

As a safe intervention, exercise, is recommended to manage FC by the clinical practice guideline which was established by an expert workgroup in the Spanish Society of Digestive Diseases (Sociedad Espanola de Patologia Digestiva) ${ }^{13-15}$ The reason is that the effectiveness and safety of exercise therapy for constipation has been recognised. ${ }^{1617}$ Among the exercise therapies, mind-body exercise has aroused widespread concern for its dual influence on the body and mind of the 
patients. For example, Taiji, a classical mind-body exercise originated from ancient China, has been shown to be effective for improving both symptoms and emotional disorder for patients with gastrointestinal dysfunction. ${ }^{1819}$

However, whether the gastrointestinal adjustment of Taiji significantly differs from the simple aerobic exercise and the potential mechanism remains uncertain. In the last two decades, the important role of the brain-gut axis in the pathogenesis of FC has been confirmed by more and more evidence. ${ }^{20}$ The dysfunction of central nervous system in processing the gastrointestinal signals has been thought to contribute to the occurrence of constipation. ${ }^{21}$ In our previous studies, we found that, compared with healthy subjects (HS), patients with FC have shown significant changes in cerebral function by functional MRI (fMRI).

So in this study, we try to (1) evaluate the clinical efficacy of simple aerobic exercise and Taiji (mind-body exercises) for treating FC with a randomised control design to investigate the differences in influencing intestinal function between aerobic exercise and Taiji practice; (2) detect the cerebral activity changes in patients with FC elicited by simple aerobic exercise and Taiji boxing using fMRI and positron emission tomography (PET)-CT to investigate the differences in influencing brain function between aerobic exercise and Taiji practice; (3) assess the difference in influence psychological state between aerobic exercise and Taiji practice; (4) analyse the potential correlations between the clinical improvements and cerebral activity changes, so as to partly explore the impossible mechanism of Taiji regulating gastrointestinal function, and provide an approach for FC treatment.

\section{METHODS AND ANALYSIS \\ Study design}

The trial is designed as a randomised control trial. A total of 80 patients with FC will be randomly assigned to the Taiji group or aerobic exercise group (1:1 ratio). The study procedure is outlined in figure 1 .

\section{Sample size calculation}

We will compare the reduction in the severity of FC symptoms between the two groups (measured by the complete spontaneous bowel movements (CSBMs)). The sample size was calculated by specifying an effect size based on minimal clinically important differences. In the case of symptoms of FC, we calculated an $80 \%$ power of detecting a conservative effect size of 0.7 . Considering $\alpha=0.05,1-$ $\beta=0.8$ and 2.0 of the SD, the study design required sample size of 68 , with a drop-out rate of $15 \%$. A total of 80 patients with FC will be finally recruited.

According to previous neuroimaging studies, ${ }^{22} 23$ 15 subjects represent a reasonable sample size for the measurement of stable cerebral responses. In this study, 15 participants in each group will be randomly chosen to undergo both MRI and PET-CT scans.

\section{Participants and recruitment strategy}

Patients who match the inclusion criteria will be recruited. They will be informed of the possible benefits and risks of exercise in the study before recruitment. And then the participants will be required to fill out informed consent and can withdraw from the study without any conditions.

\section{Inclusion criteria}

Patients will be included if they: (1) meet the Rome IV Diagnostic Criteria for FC which was designed in 2016 (online supplementary material 1); (2) are right-hand and aged 18-35 years; (3) has less than three CSBMs per week; (4) get more than 10 scores when are evaluated by Cleveland Constipation Score (CCS) and (5) provide written informed consent.

\section{Exclusion criteria}

Patients will be excluded if they (1) are pregnant, or intend to pregnant in 3 months, or be lactating; (2) are not physically fit for exercise; (3) have a history of head trauma and loss of consciousness; (3) have diabetes or serious cardiovascular, neurological, psychiatric, renal or respiratory disease; (4) have moderate or above symptoms of depression and anxiety; (5) cannot maintain silence in a lying position for $20 \mathrm{~min}$; (6) have any contraindications to fMRI scanning including the presence of metal stent, metal denture or claustrophobia; (7) have participated in other exercises that may improve constipation symptoms (including meditation and yoga) over $30 \mathrm{~min}$ per week in the last 3 months; (8) have taken gastrointestinal dynamic medicine, non-steroidal anti-inflammatory drugs and steroids during the last 15 days; or have taken traditional treatments (including surgery, diet modification, biofeedback or probiotics) in the previous 1 month and (9) have participated in any other clinical trials in the past 3 months.

\section{Recruitment strategy}

Participants with FC will be recruited from the First Affiliated Hospital of Chengdu University of Traditional Chinese Medicine (TCM) and the campus of Chengdu University of TCM by advertisement from December 2018 to November 2020.

\section{Randomisation and blinding}

Using a computer-generated randomisation digital table. The randomisation will entail in two steps: (1) 80 patients will be equally allocated into the two treatment arms; (2) 15 patients will be randomly chosen from each group to receive fMRI scans and PET-CT scans. We will use opaque, sealed envelopes to conceal the sequence until interventions are assigned.

It is difficult to blind instructors and patients in this trial for different interventions. However, outcome assessors and statistical analysts will be blind to the procedure and result of randomisation, group allocation and intervention. 


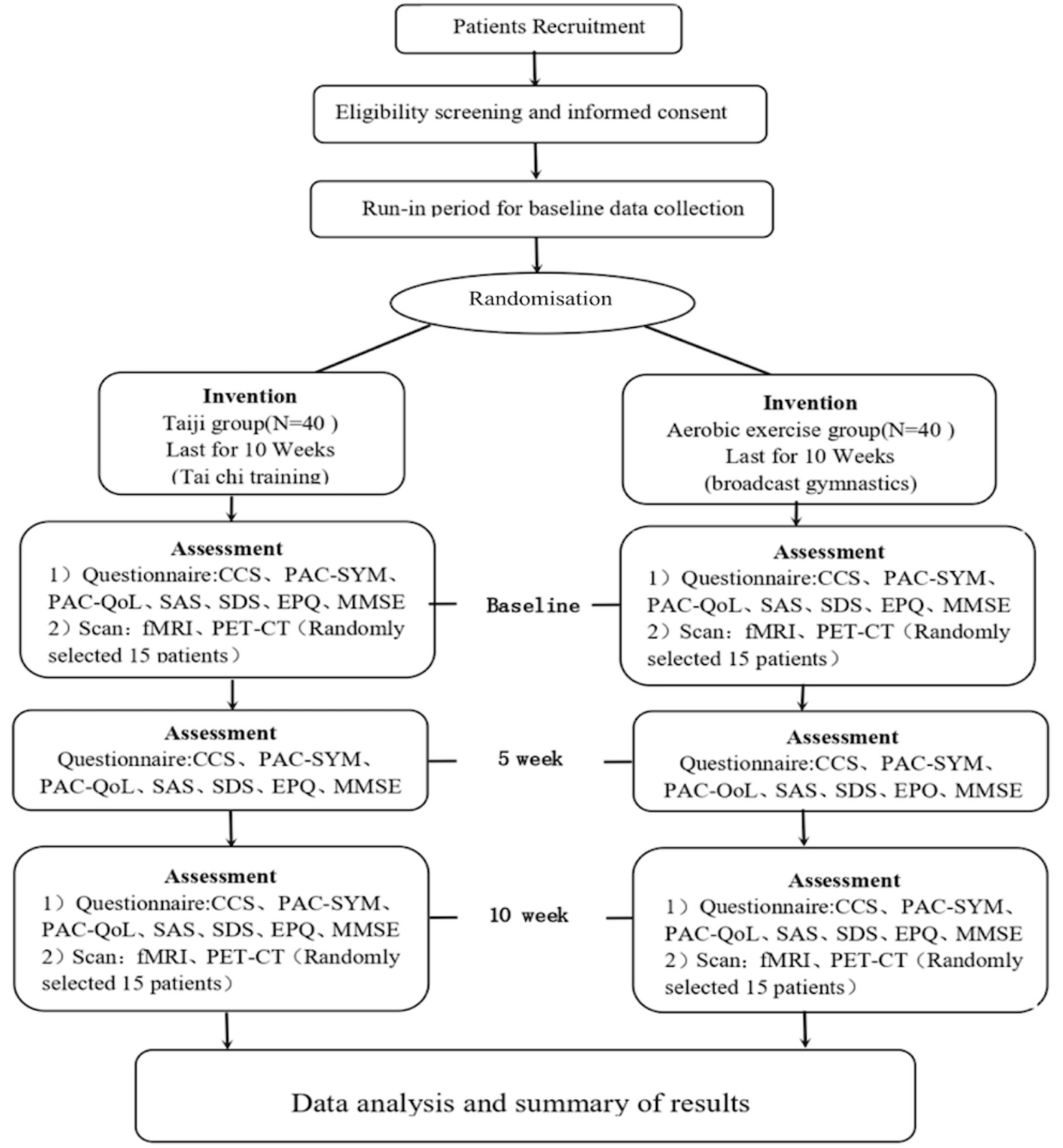

Figure 1 Flowchart of the participants (procedure) through the trial.

\section{Patient and public involvement}

During the development of the study protocol, some patients were interviewed about the acceptability of taking photo or video in the procedure, and they supported the study and expressed no concerns regarding the photo or video. The patients are asked if they would like to receive a copy of the outcomes of the study after the invention and an email address or telephone number are collected to facilitate the distribution of any relevant publications. The patients will be informed of the result of this study after the intervention. Results will be disseminated through policy briefs, workshops, peer-reviewed publications and conferences.

\section{Intervention}

Taiji group

Because none of the participants in Taiji group had practiced Taiji, they will undergo a 5-day training session with 24-style Yang's Taiji (online supplementary material 2), and be required to master the movement essentials of Taiji before the formal trial.

The formal Taiji practice will be performed four times a week (Monday, Tuesday, Thursday and Friday) and last for 10 weeks. Each practice session takes $60 \mathrm{~min}$ and includes 10- min warm-ups, $40 \mathrm{~min}$ Taiji practice and 10 min relaxation. 


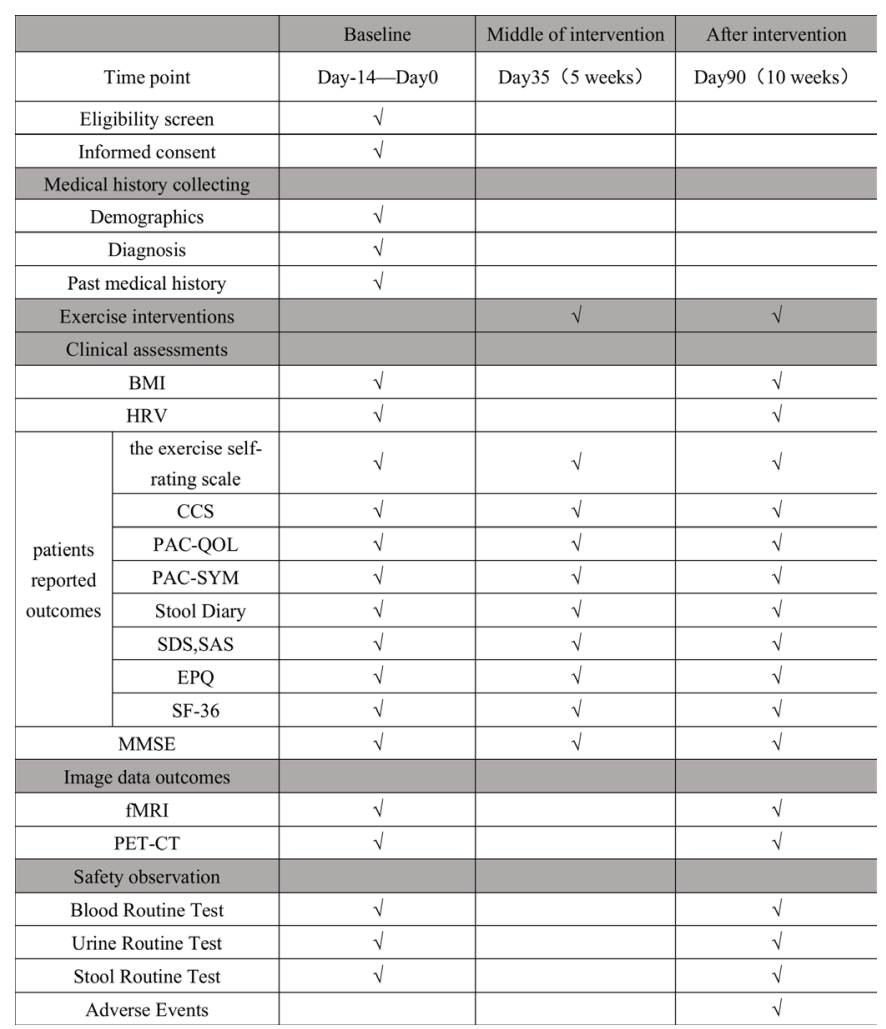

Figure 2 Time schedule of enrollment, interventions and assessments: at the baseline, the median of intervention (5-week exercise) and the end of intervention (10-week exercise), the stool diary, Cleveland Constipation Score (CCS) and Patient Assessment of Constipation Symptom (PACSYM) will be used to evaluate the clinical efficacy of different interventions; the Patient Assessment of Constipation Quality of Life Questionnaire (PAC-QoL) will be used to assess the health-related QoL; the Self-rating Depression Scale (SDS), Self-rating Anxiety Scale (SAS), Eysenck Personality Questionnaires (EPQ) and Mini-Mental State Examinations (MMSE) will be used to consider the effect of psychological factors on the patients symptoms. All participants will be assessed the 24 hours heart rate variability (HRV) to evaluate the autonomic nervous function at the baseline and the end of the10-week intervention. Functional MRI scans and positron emission tomography-CT scans will be performed to detect the cerebral functional changes in 15 patients in each group both at the baseline and at the end of the intervention.

The 24-style Yang's Taiji will be trained by two professional Taiji instructors with over 10 years of training experience. To ensure correct posture and speed of movement, all sessions will be monitored by regular reviews of video recordings and feedback sessions to ensure correct instructions.

\section{Aerobic exercise group}

In the aerobic exercise group, patients will undergo the broadcast gymnastics (online supplementary material 3) for 10 weeks. As same as Taiji group, they will undergo a 5-day training session with broadcast gymnastics, and be required to master the movement essentials of broadcast gymnastics before the formal trial.
The formal broadcast gymnastics practice will be performed four times a week (Monday, Tuesday, Thursday and Friday) and last for 10 weeks. Each practice session takes $60 \mathrm{~min}$, including breathing, warm-ups, stretching movement training and relaxation.

To ensure correct posture and speed of movement, the broadcast gymnastics will be trained by two professional instructors, and all sessions will be monitored by regular reviews of video recordings and feedback sessions.

\section{Concomitant medications}

If the constipation symptom of participants gets worse and interfere with daily life, or less than two bowel movements per week, the investigators will administer the Glycerine Enemas as a necessary concomitant medication. And the concomitant medications will be detailed and recorded in case report forms.

\section{MEASUREMENTS}

The measurements will be drawn from independent assessors who have been trained prior to the study. All outcomes will be recorded regardless of whether the participants complete the research programme. The schedule of the study, including enrollment, interventions, assessments and visits for participants, is shown in figure 2.

\section{General information collection}

General information includes the demographic data and vital sign. The demographic data include age, gender, level of education, nationality and BMI. The vital sign includes body temperature, heart rate, respiratory rate and blood pressure. Among them, the heart rate of each patient will be immediately recorded after every training.

\section{Exercise evaluation}

After each training, the exercise self-rating scale from the American College of Sports Medicine will be filled by all participants to evaluate exercise intensity scoring and grading that can control the levels of exercise both objectively and subjectively to obtain the exercise requirement.

\section{Symptoms measurement}

The following scales will be used to evaluate the constipation symptoms of patients at the baseline, the median of intervention (5-week exercise) and the end of intervention (10-weeks' exercise).

\section{The stool diary}

It consists of seven sections: (1) weekly CSBMs; (2) the Bristol Stool Form Scale ${ }^{24}$ containing seven types of stool evacuation; (3) straining; (4) defecating time; (5) incomplete feeling; (6) unsuccessful defecation frequency in 24 hours and (7) anus fall bilge feeling.

\section{Cleveland Constipation Score}

CCS is a designated scale to evaluate patients' gastrointestinal or anorectal symptoms. $\mathrm{CCS}^{25}$ will be formulated by 
the American Gastroenterology Society, including eight aspects of the constipation symptoms. The higher score obtained by the participants indicating the more serious the degree of constipation.

\section{Patient Assessment of Constipation Symptoms}

Patient Assessment of Constipation Symptom (PAC-SYM) is also to evaluate patients with gastrointestinal symptoms. PAC-SYM consists of 12 items of faecal traits, rectal symptoms and abdominal symptoms. Each item is scored from 0 to 4 , a higher score indicating more severe symptoms.

\section{Measurements for the quality of life}

The following two questionnaires will be used to evaluate the QoL of patients at the baseline, the median of intervention (5-week exercise) and the end of intervention (10-week exercise).

\section{Patient Assessment of Constipation Quality of Life Questionnaire}

Patient Assessment of Constipation Quality of Life Questionnaire (PAC-QoL) ${ }^{26}$ is an internal consistency reliability scale consisting of 28 items namely, body discomfort, psychological discomfort, worries and concerns, and satisfaction. It used to assess the quality of life of patients with FC.

\section{Psychological state assessment}

Considering the effect of psychological factors on the patients with symptoms, the Self-rating Depression Scale (SDS), ${ }^{27}$ the Self-rating Anxiety Scale (SAS) ${ }^{28}$ the Eysenck Personality Questionnaires (EPQ ${ }^{29}$ and the Mini-Mental State Examinations (MMSE) ${ }^{30}$ will be performed on all the patients at the baseline, the median of intervention (5-week exercise) and the end of intervention (10-week exercise).

\section{Evaluation of autonomic nervous function}

Because the autonomic nervous plays an important role in regulating intestinal function, ${ }^{31} 32$ this study selected the heart rate variability (HRV) to evaluate the autonomic nervous function. HRV is a sensitive, quantitative and intuitive indicator for non-invasive assessment of autonomic nervous activity. ${ }^{33}$ All participants will be assessed during the 24-hour HRV at the baseline and the end of the 10 -week intervention. At least 12 hours prior in the examination period, medication (including FC medications), tobacco, alcohol, caffeine, tea or other stimulating beverages will be forbidden. Data collection will begin at 17:00 and conclude at 17:00 the next day. During the 24-hour period, participants will be allowed sufficient sleep and will avoid strenuous exercise and emotional fluctuations. The device (CT-086S, BENE WARE, CHINA) will capture the $\mathrm{R}-\mathrm{R}$ intervals through the attachment of electrodes to the chest.

\section{Neuroimaging scans}

A total of 15 participants in each group will receive both MRI scans and PET-CT scans at the baseline and the end of the intervention.

\section{MRI scan}

The tridimensional structural image scanning parameters will be as follows: repetition time/echo time $=1900$ $\mathrm{ms} / 2.26 \mathrm{~ms}$; slice thickness $=1 \mathrm{~mm}$; slice number $=30$; matrix size $=128 \times 128$ and the field of view $=256 \times 256 \mathrm{~mm}$. The Blood Oxygenation Level-Dependent fMRI (BOLDfMRI) scanning parameters will be as follows: repetition time $/$ echo time $=2000 \mathrm{~ms} / 30 \mathrm{~ms}$; flip angle $=90^{\circ}$; slice number $=30$; matrix size $=128 \times 128$; field of view $=240 \times$ $240 \mathrm{~mm}$, slice thickness $=5 \mathrm{~mm}$; total volume $=240$. Diffusion Tensor Imaging (DTI) data will be acquired using a single-shot spin-echo echo planar image sequence with the following parameters: field of view $=240 \times 240$ $\mathrm{mm}$; repetition time $/$ echo time $=6800 \mathrm{~ms} / 93 \mathrm{~ms}$; matrix size $=128 \times 128$; slice thickness $=3 \mathrm{~mm}$ with no gap. Two diffusion-weighted sequences will be acquired using gradient values $b=1000 \mathrm{~s} / \mathrm{mm}^{2}$ and $b=0$, with the diffusion-sensitising gradients applied in 30 non-collinear directions.

\section{ositron emission tomography-CT scan}

The PET-CT images will be initially acquired using the following parameters: $130 \mathrm{KV}$; $100 \mathrm{mAs}$; matrix size $=256 \times 256$; magnification $=2 \times$; slice thickness $=5 \mathrm{~mm}$; slice interval $=2.5 \mathrm{~mm}$; pitch $=9.2 \mathrm{~mm}$. PET will then be performed using the following parameters: $\operatorname{bed}=1$, model $=3 \mathrm{D}$, scanning time $=8 \mathrm{~min}$ and total counts $=3 \times 10^{9}$. Following data acquisition, images will be reconstructed using ordered-subset expectation maximisation with four iterations and eight subsets.

\section{Safety assessments}

Participants will be monitored during the study intervention for the occurrence of adverse events. Adverse events associated with this study will occur during the exercise period or during neuroimaging data scanning. All adverse events will be detailed and recorded on an adverse event case report form during the study intervention. All events will be evaluated for their relevance to the intervention and severity, according to the gastroenterology clinician.

\section{Statistical analysis \\ Clinical data analysis}

Clinical data will be analysed by an independent statistician blinded to the test settings using SPSS V.20.0 statistical software. Data analysis will be based on the intention-totreat (ITT) and per protocol (PP) principles. The results of ITT and PP analysis will be compared for consistency. A $p$ value $<0.05$ will be considered statistically significant. Participants who fail to complete the study will be treated as having no change from baseline at all times. All data will be presented as the mean $\pm \mathrm{SD}$. Categorical variables will be described with percentages (\%). Clinical data among the two groups will be compared using a two-sample t-test (Taiji group vs aerobic exercise group), and comparisons between baseline and the end of treatment in each group will be performed using a paired sample t-test. Clinical data on the skewed distribution will be compared 
using a non-parametric test. Categorical variables will be compared using a $\chi^{2}$ test or Fisher's exact test.

\section{Neuroimaging data analysis}

For fMRI scans, all preprocessing steps will be performed using DPARSF software ( http://rfmri.org/DPARSF ) based on MATLAB. The main analytical methods for the cerebral responses of the different interventions will contain regional homogeneity, an amplitude of low-frequency fluctuation (ALFF) and functional connectivity. Following data preprocessing, data analysis will consist of two stages: (1) whole-brain performance analysis through ALFF and (2) functional connectivity through seed correlation analysis. In this study, the cerebral responses to Taiji in FC mainly refer to a change in the ALFFs and functional connectivity before and after treatment. A threshold of voxel-wise uncorrected $\mathrm{p}<0.005$ and $\mathrm{p}<0.05$ familywise error correction at the cluster level will be applied to all analyses.

SPM12 software (http://www.fil.ion.ucl.ac.uk/spm) will be used to process and analyse the PET-CT data with MATLAB 2015b. Regional brain radioactivity will be measured to assess regional cerebral glucose metabolism which directly reflects the cerebral activity.

Correlation analysis will be measured using the Pearson correlation coefficient using clinical and fMRI imaging data.

\section{DISCUSSION}

This study is the first to investigate the differences in regulation brain-gut balance between Taiji and simply aerobic exercise for patients with FC. It provides a potential therapy for clinical treatment of FC, and a new approach to investigate mind-body exercises from clinical symptoms, mental state and brain function.

\section{The multidimensional evaluation of Taiji for treating FC}

Taiji is a widely spread mind-body exercise. It has been thought of as 'moving meditation' by the National Institute of Health for its significant physical and psychological benefit. For example, it has found that comparing to aerobic exercise, Taiji as a mind-body exercise could result in similar improvement in symptoms of fibromyalgia, but greater improvement in several secondary outcomes (such as global assessment of patients with fibromyalgia; anxiety and self-efficacy and coping strategies) ${ }^{34}$ However, there is no direct evidence to prove the therapeutic benefit of Taiji to FC. When we want to objectively evaluate the Taiji's effect on FC, we must take into account its influence on physical aspects (constipation symptoms and QoL) and psychological aspect (emotional disorder).

Furthermore, FC is a typical psychosomatic disease, the gastrointestinal factors and psychological factors play important role in the onset of this disorders, and the interaction of gastrointestinal factors and psychological factor makes FC recurrent and persistent. It is reported that patients with FC often have depression and anxiety, and the emotion can reverse to aggravate the symptoms of constipation. ${ }^{13}$ Moreover, more and more studies had proven that cerebral structural and functional abnormalities participated in the pathogenesis of FGIDs including FC. Comparing to HS, patients with FC have shown altered activities in emotional process modulation (dorsal anterior cingulate cortex, anterior insula, orbitofrontal cortex and hippocampus), somatic and sensory processing, and motor control (supplementary motor area and precentral gyrus).$^{35}$ Therefore, the improvements of symptoms, QoL, emotional disorder and the changes of brain function must be considered when evaluating the therapeutic effect of FC.

Therefore, the assessment of Taiji for treating FC should be a multidimensional evaluation. In this study, we try to evaluate the therapeutic effect of Taiji forms the following five aspects. First, the stool diary, CCS and PAC-SYM will be used to assess the clinical symptoms. The stool diary is the self-rating recording for the clinical symptoms changes, especially the CSBM includes the patient's perception of satisfactory evacuation. For evaluating patients with gastrointestinal or anorectal symptoms, the CCS (a selfrating scale) and the PAC-SYM (an other-rating scale) will be used simultaneously. Second, the PAC-QoL is the constipation-specific questionnaire and will be used to more completely evaluate the improvements of QoL. Thirdly, SDS, SAS, EPQ and MMSE will be performed to assess the emotional and cognitive state of patients with FC. The former two are self-rating scales, and the latter two are other rating scales. Fourthly, HRV is used to evaluate the autonomic nervous function. Finally, the most commonly used neuroimaging techniques, MRI scans and PET-CT scans, will be performed to investigating the cerebral activity changes elicited by Taiji and acerbic exercise. The collaborative application of fMRI and PET-CT can define functional images with high resolution and provide biological information on molecular metabolism with high sensitivity to keep high data reproducibility. ${ }^{36}$ Taken together, the multidimensional evaluation system is more in line with the therapeutic characteristics of Taiji and the pathogenic features of FC and will help to assess the effect of Taiji for FC more comprehensively.

\section{The quality control criteria}

To improve the reliability and reproducibility of the results, stricter quality control criteria will be designed and performed in this study.

For the participation enrollment, the strict Inclusion and Exclusion Criteria is established to improve the homogeneity of the baseline values. We will only include the participants who are naive to Taiji and have not taken other exercise that may improve constipation symptoms (including meditation and yoga).

For the Taiji and aerobic exercises, we have established the standard training procedures for Taiji and aerobic-exercises practice including the movements essential, training time and frequency. To ensure the 
standardisation of movements, the training of Taiji and aerobic-exercises will be completed by two trained coaches who have performed these exercises for over 10 years. In subsequent training sessions, researchers will video-record each session and the coaches will randomly check the standardisation of the movements.

For the multimode neuroimaging scans, we have established a realistic standard operating procedure for multimode neuroimaging scans including the imaging data acquisition and fusion. To consider the dynamic variability of brain function, the acquisition of imaging data will be held with the same scanner and operator in the morning of the same day. Moreover, participants will be asked to maintain their regular lifestyle and avoid overexertion, staying up late and ingesting alcohol, tobacco, tea and coffee, in order to keep the stability of physiological and psychological status. Before the scan, each participant's emotional state will be assessed by the emotional state assessment scale. The temperature of scanning room will be kept at $18-22^{\circ} \mathrm{C}$,with humidity more than $60 \%$ and noise less than $150 \mathrm{~dB}$; to ensure reliability of multimodal imaging data analysis, the BOLD-MRI and PET-CT data will be collected separately and be interpreted in the same template space to cross-verify the results.

For the measurement, first the researchers, exercise instructors and outcome assessors are separated in the study process. Second, the physical and mental status of participants will be evaluated by the same clinical physician and psychological experts, respectively. Thirdly the blind method will be used for data statistics and analysis to avoid the measurement bias. The whole study design will be concealed to the outcome assessors.

In conclusion, Taiji has been shown to be effective for improving both symptoms and emotional disorder for patients with gastrointestinal dysfunction. This trial is the first randomised controlled neuroimaging trial to investigate the differences in influencing intestinal function between simple aerobic exercise and Taiji practice. The results will demonstrate the differences in regulation brain-gut balance between Taiji and simply aerobic exercise for patients with $\mathrm{FC}$ and provide a potential therapy for clinical treatment of FC, and a new approach for the research of mind-body exercise.

\section{Trial status}

This trial is currently in the stage of recruiting patients. The first patient was included on 1 December 2018. To date, 23 patients have been included.

\author{
Author affiliations \\ ${ }^{1}$ Acupuncture and Tuina School/The 3rd Teaching Hospital, Chengdu University of \\ Traditional Chinese Medicine, Chengdu, Sichuan, China \\ ${ }^{2}$ School of Gymnasium, Chengdu University of Traditional Chinese Medicine, \\ Chengdu, Sichuan, China \\ ${ }^{3}$ School of Chinese Classics, Chengdu University of Traditional Chinese Medicine, \\ Chengdu, Sichuan, China \\ ${ }^{4}$ Chengdu University of Traditional Chinese Medicine, Chengdu, Sichuan, China
}

Acknowledgements We thank Xueling Suo and Kun Qin, Huaxi MR Research Center, Departments of Radiology, West China Hospital of Sichuan University for the instruction of fMRI scanning parameters. And We also thank Hongjun Xie, PET-CT Research Center, Departments of Radiology, Sichuan Provincial People's Hospital for the instruction of PET-CT scanning parameters. We also thank Jianwei Wu and Ning Liu for providing a schematic diagram of the movements of Taiji and broadcast gymnastics

Collaborators Xueling Suo,Kun Qin, Huaxi MR Research Center, Departments of Radiology, West China Hospital of Sichuan University for the instruction of fMRI scanning parameters ; Hongjun Xie, PET-CT Research Center, Departments of Radiology, Sichuan Provincial People's Hospital for the instruction of PET-CT scanning parameters.

Contributors SY, JC, YG, YT, FZ and S-GY, Acupuncture and Tuina School/The 3rd Teaching Hospital, Chengdu University of Traditional Chinese Medicine, conceived and designed the study. JW and TL, School of Gymnasium, Chengdu University of Traditional Chinese Medicine, designed the training protocol of Taiji and aerobic exercise. S-GY, JC and YG participated in drafting the trial protocol and preparing the manuscript. YT, RY and ZH, Acupuncture and Tuina School/The 3rd Teaching Hospital, Chengdu University of Traditional Chinese Medicine, participated in data collection and were in charge of recruitment and treatment of patients. All authors read and approved the final manuscript.

Funding The trial is financially supported by the National Special Support Program For High-level Personnel Recruitment (W02020595).

Competing interests None declared.

Patient consent for publication Obtained.

Ethics approval The procedures have been approved by the Sichuan Regional Ethics Review Committee on Traditional Chinese Medicine (No. 2018KL-047) and conformed to the Declaration of Helsinki. Results will be disseminated through policy briefs, workshops, peer-reviewed publications and conferences.

Provenance and peer review Not commissioned; externally peer reviewed.

Open access This is an open access article distributed in accordance with the Creative Commons Attribution Non Commercial (CC BY-NC 4.0) license, which permits others to distribute, remix, adapt, build upon this work non-commercially, and license their derivative works on different terms, provided the original work is properly cited, appropriate credit is given, any changes made indicated, and the use is non-commercial. See: http://creativecommons.org/licenses/by-nc/4.0/.

\section{REFERENCES}

1. Drossman DA. The functional gastrointestinal disorders and the Rome III process. Gastroenterology 2006;130:1377-90.

2. Zhao $Y-F$, Ma X-Q, Wang R, et al. Epidemiology of functional constipation and comparison with constipation-predominant irritable bowel syndrome: the systematic investigation of gastrointestinal diseases in China (SILC). Aliment Pharmacol Ther 2011;34:1020-9.

3. Mugie SM, Benninga MA, Di Lorenzo C. Epidemiology of constipation in children and adults: a systematic review. Best Pract Res Clin Gastroenterol 2011;25:3-18.

4. Rajindrajith S, Devanarayana NM. Constipation in children: novel insight into epidemiology, pathophysiology and management. $J$ Neurogastroenterol Motil 2011;17:35-47.

5. Zhou H, Yao M, Cheng G-Y, et al. Prevalence and associated factors of functional gastrointestinal disorders and bowel habits in Chinese adolescents: a school-based study. J Pediatr Gastroenterol Nutr 2011;53:168-73.

6. Boronat AC, Ferreira-Maia AP, Matijasevich A, et al. Epidemiology of functional gastrointestinal disorders in children and adolescents: a systematic review. World J Gastroenterol 2017;23:3915-27.

7. Pare P, Ferrazzi S, Thompson WG, et al. An epidemiological survey of constipation in Canada: definitions, rates, demographics, and predictors of health care seeking. Am J Gastroenterol 2001;96:3130-7.

8. Sun SX, Dibonaventura M, Purayidathil FW, et al. Impact of chronic constipation on health-related quality of life, work productivity, and healthcare resource use: an analysis of the National health and wellness survey. Dig Dis Sci 2011;56:2688-95.

9. Serrano-Falcón B, Rey E. The safety of available treatments for chronic constipation. Expert Opin Drug Saf 2017;16:1243-53.

10. Dipalma JA, Cleveland MV, McGowan J, et al. A randomized, multicenter, placebo-controlled trial of polyethylene glycol laxative for chronic treatment of chronic constipation. Am J Gastroenterol 2007;102:1436-41.

11. Zhang C, Guo L, Guo X, et al. Short and long-term efficacy of combining Fuzhengliqi mixture with acupuncture in treatment of functional constipation. J Tradit Chin Med 2013;33:51-9. 
12. Li Y, An L, Tian H. [Diabetic constipation treated with acupoint embedding therapy and forlax: a randomized controlled trial]. Zhongguo Zhen Jiu 2016;36:124-8.

13. Mearin F, Ciriza C, Mínguez M, et al. Clinical practice guideline: irritable bowel syndrome with constipation and functional constipation in the adult. Rev Esp Enferm Dig 2016;108:332-63.

14. Silva CAG, Motta MEFA. The use of abdominal muscle training, breathing exercises and abdominal massage to treat paediatric chronic functional constipation. Colorectal Dis 2013;15:e250-5.

15. Costa RJS, Snipe RMJ, Kitic CM, et al. Systematic review: exerciseinduced gastrointestinal syndrome-implications for health and intestinal disease. Aliment Pharmacol Ther 2017;46:246-65.

16. Schumann D, Anheyer D, Lauche R, et al. Effect of Yoga in the Therapy of Irritable Bowel Syndrome: A Systematic Review. Clin Gastroenterol Hepatol 2016;14:1720-31.

17. Silva LMT, Cignolini A, Warren R, et al. Improvement in sensory impairment and social interaction in young children with autism following treatment with an original Qigong massage methodology. Am J Chin Med 2007;35:393-406.

18. Birdee GS, Cai H, Xiang Y-B, et al. T'ai chi as exercise among middle-aged and elderly Chinese in urban China. J Altern Complement Med 2013;19:550-7.

19. Zhou C, Zhao E, Li Y, et al. Exercise therapy of patients with irritable bowel syndrome: a systematic review of randomized controlled trials. Neurogastroenterol Motil 2019;31:e13461.

20. Mayer EA, Aziz Q, Coen S, et al. Brain imaging approaches to the study of functional Gi disorders: a Rome working team report. Neurogastroenterol Motil 2009;21:579-96.

21. Equit M, Niemczyk J, Kluth A, et al. Central nervous system processing of emotions in children with fecal incontinence and constipation. Z Kinder Jugendpsychiatr Psychother 2019;47:67-71.

22. Desmond JE, Glover GH. Estimating sample size in functional MRI (fMRI) neuroimaging studies: statistical power analyses. J Neurosci Methods 2002;118:115-28.
23. Hayasaka S, Peiffer AM, Hugenschmidt CE, et al. Power and sample size calculation for neuroimaging studies by non-central random field theory. Neuroimage 2007;37:721-30.

24. Lewis SJ, Heaton KW. Stool form scale as a useful guide to intestinal transit time. Scand J Gastroenterol 1997;32:920-4.

25. Agachan F, Chen T, Pfeifer J, et al. A constipation scoring system to simplify evaluation and management of constipated patients. Dis Colon Rectum 1996;39:681-5.

26. Marquis P, De La Loge C, Dubois D, et al. Development and validation of the patient assessment of constipation quality of life questionnaire. Scand J Gastroenterol 2005;40:540-51.

27. ZUNG WW. A self-rating depression scale. Arch Gen Psychiatry 1965;12:63-70.

28. Zung WW. A rating instrument for anxiety disorders. Psychosomatics 1971;12:371-9.

29. Schuldberg D. Eysenck personality questionnaire scales and paperand-pencil tests related to creativity. Psychol Rep 2005;97:180-2.

30. Creavin ST, Wisniewski S, Noel-Storr AH, et al. Mini-Mental state examination (MMSE) for the detection of dementia in clinically unevaluated people aged 65 and over in community and primary care populations. Cochrane Database Syst Rev 2016;25

31. Tougas $\mathrm{G}$. The autonomic nervous system in functional bowel disorders. Gut 2000;47(90004):78iv-80.

32. Knowles $\mathrm{CH}$, Scott SM, Lunniss PJ. Slow transit constipation: a disorder of pelvic autonomic nerves? Dig Dis Sci 2001;46:389-401.

33. Patel HC, Rosen SD, Lindsay A, et al. Targeting the autonomic nervous system: measuring autonomic function and novel devices for heart failure management. Int J Cardiol 2013;170:107-17.

34. Wang C, Schmid CH, Fielding RA, et al. Effect of tai chi versus aerobic exercise for fibromyalgia: comparative effectiveness randomized controlled trial. BMJ 2018;360:k851.

35. Zhu Q. Weiwei Cai,Jianyong Zheng,et al.Distinct resting-state brain activity in patients with functional constipation. Neurosci Lett 2016;632:141-6.

36. Li F, Harmer P, Fitzgerald K, et al. Tai chi and postural stability in patients with Parkinson's disease. N Engl J Med 2012;366:511-9. 